\title{
On the Precipitation of Hydrides in Metals under Stress - Finite Deforma- tion Approach
}

\author{
Andreas G. Varias* \\ Ministry of Defense, Hallenic Navy, Athens, Greece
}

\begin{abstract}
An analytic relation for the terminal solid solubility of hydrogen in a stressed metal is derived, based on finite deformation theory. Phase transformation is assumed to be a reversible process, which occurs under local chemical equilibrium among hydride, metal and hydrogen in solid solution. Hydrogen terminal solid solubility depends on stress due to the interaction of the applied stress field with the field of the expanding hydride as well as due to the reduction of hydrogen chemical potential in solid solution, caused by hydrostatic stress. The present analysis is complementary to hydrideinduced embrittlement studies in metals, under conditions, which require the use of finite deformations.
\end{abstract}

Keywords: Terminal solid solubility, hydrogen, hydride.

\section{INTRODUCTION}

Hydride-induced embrittlement is one type of material deterioration caused by hydrogen in metals such as zirconium, titanium, niobium and vanadium. According to in-situ electron microscopy studies (e.g. [1-4]), embrittlement occurs by precipitation of brittle hydrides at stress concentration locations. Indeed, a positive hydrostatic stress reduces hydrogen chemical potential in solid solution [5], thus creating hydrogen flux towards stress concentrators. When hydrogen terminal solid solubility is reached at the stress concentration locations, hydrides precipitate. Subsequently crack generation and propagation may occur by hydride fracture.

An important part, in predicting hydride-induced fracture, is the determination of hydrogen terminal solid solubility. Earlier theoretical studies have shown that terminal solid solubility depends on stress. In [6], the effect of stress on hydrogen terminal solid solubility is introduced by hydride expansion during precipitation. An additional stress effect was revealed in [7], caused by the reduction of hydrogen chemical potential in solid solution due to hydrostatic stress. Recently in [8, 9], hydrogen terminal solid solubility was derived analytically for anisotropic hydrides of any shape with different elastic properties than those of the metal, assumed to be anisotropic too. According to relations (9a-b) in [9], a third stress effect on terminal solid solubility is attributed to the change of elastic material properties, when phase transformation occurs.

All previously mentioned analytical relations for hydrogen terminal solid solubility were derived, based on infinitesimal displacement gradient theory. However, in several applications, at room or higher temperatures, crack growth occurs by repeating steps, which involve crack tip blunting, hydride precipitation at a distance from the crack tip, hydride

*Address correspondence to this author at the Ministry of Defense, Hallenic Navy, Athens, Greece; Tel: +30-210-2821655; Fax: +30-210-2849916; E-mail: smro@hol.gr fracture and ductile rupture of the ligament between the main crack and the generated micro-crack (e.g. [10, 11]). This mechanism is also called delayed hydride cracking. Crack tip blunting as well as ligament rupture are associated with significant material deformation (e.g. fig. 3-7 in [11]). Therefore, a finite deformation approach is necessary for the detailed simulation of a crack growth step, which involves the simultaneous operation of the coupled processes of hydrogen diffusion, hydride precipitation, non-mechanical energy flow, mainly in the presence of temperature gradient as in nuclear industry applications, and material deformation. In previous papers, the governing equations for mass diffusion and non-mechanical energy flow in metals, under finite deformation, were presented $[12,13]$. In the present work, the terminal solid solubility of hydrogen in a metal is derived by employing finite deformations. Thus, a tool for predicting hydride precipitation is provided. In other words, the present relation of hydrogen terminal solid solubility, together with the governing equations for hydrogen diffusion and nonmechanical energy flow in $[12,13]$, can be part of a finite element algorithm, based on finite deformation, for the detailed simulation of delayed hydride cracking.

Hydride precipitation in a metal is generally associated with elastic as well as plastic deformation of the metal. In vanadium, at relatively low temperatures, hydride accommodation is elastic. In zirconium, plastic deformation occurs at room or larger temperatures. Hydride size is also a factor, affecting the type of deformation during precipitation. In the case of coherent sub-micron precipitates, the effective yield stress becomes equal to the theoretical yield strength and thus plastic relaxation is not possible unless the transformation stress, caused by the misfitting precipitate, is extremely large [14]. For incoherent sub-micron precipitates, the effective yield stress is approximately inversely proportional to the precipitate size and plastic relaxation is again impossible for precipitate size less than $10 \mathrm{~nm}$. Note that the occurrence, or not, of plastic deformation during precipitation has important implications on hydride stability. In vanadium, with elastic hydride accommodation at relatively low temperatures, the hydrides, which form ahead of the crack tip, re- 
dissolve after fracture and crack propagation [1]. On the other hand, in zirconium alloys at room or larger temperatures, hydrides are found on the crack faces [11].

According to the above discussion, the precipitation of a sub-micron hydride, which corresponds to a slight increase of hydride volume fraction in a metal, is accommodated elastically. However, the growth of the hydrides may eventually lead to irreversible metal plastic deformation, depending on temperature and hydride expansion during precipitation. This effect could be taken into account in a numerical simulation, by elastic-plastic governing equations for the deformation of the metal. Such an approach has been successfully followed in previous theoretical studies $[9,15]$, under infinitesimal displacement gradient theory, which show excellent agreement with experimental measurements of threshold stressintensity factor and steady-state sub-critical crack growth velocity; these studies do not simulate the details of a crack growth step. Having in mind the cases, where hydride accommodation is elastic, as well as the approach followed in $[9,15]$, the present derivation of hydrogen terminal solid solubility is based on finite elasticity. Thus an analytic relation is derived, which clearly reveals the effect of important physical parameters and it is easily incorporated in numerical algorithms. A finite element model, based on elastic-plastic finite deformation approach, could incorporate the present terminal solid solubility relation and still simulate delayed hydride cracking accurately, when a small time step time integration scheme is employed. Indeed, within a sufficiently small time integration interval, the increase of hydride volume fraction is accommodated elastically, corresponding to the precipitation of sub-micron hydrides.

In the following, tensor notation is used throughout. Bold-faced symbols are used to denote vectors and second or higher order tensors. Products are indicated with dots and products containing no dots are dyadic products. Latin indices range from one to three and repeated Latin indices are always summed. Inverses, transposes and transposed inverses are denoted with a superscripted $-1, T$ and $-T$, respectively. For example:

$$
\begin{aligned}
& \mathbf{A} \cdot \mathbf{B}=A_{i k} B_{k j} \mathbf{b}_{i} \mathbf{b}_{j}, \\
& \mathbf{A}: \mathbf{B}=A_{i j} B_{j i}, \\
& \mathbf{c d}=c_{i} d_{j} \mathbf{b}_{i} \mathbf{b}_{j}, \\
& \mathbf{c} \cdot \mathbf{d}=c_{i} d_{i}, \\
& \mathbf{B} \cdot \mathbf{c}=B_{i k} c_{k} \mathbf{b}_{i} .
\end{aligned}
$$

The base vectors, $\mathbf{b}_{i}$, are Cartesian and independent of time, $t$.

\section{HYDROGEN TERMINAL SOLID SOLUBILITY}

A hydride forming metal, which contains hydrogen, is subjected to an externally applied load. It is assumed that the metal undergoes a purely elastic deformation, described by the deformation gradient $\mathbf{F}^{a}(=\partial \mathbf{x} / \partial \mathbf{X}) ; \mathbf{x}$ is the position of a solid particle in the deformed configuration (spatial coordinates) and $\mathbf{X}$ is its position in a reference (non-deformed) configuration (material coordinates), at $t=0$. The resulting stress field is given by Cauchy stress, $\sigma^{a}$, defined on the deformed configuration. The respective second PiolaKirchhoff stress, defined on the reference configuration, is equal to $\mathbf{S}^{a}=\left|\mathbf{F}^{a}\right| \mathbf{F}^{a-1} \cdot \sigma^{a} \cdot \mathbf{F}^{a-T}$, where $\left|\mathbf{F}^{a}\right|$ is the determinant of the deformation gradient. When the metal deforms plastically too, then $\mathbf{F}^{a}$ is set equal to the elastic part of the deformation gradient (e.g. [13]) and the reference configuration is produced by applying the mapping $\mathbf{F}^{a-1}$ on the deformed configuration, where $\sigma^{a}$ is applied.

Hydride precipitation in the stressed metal occurs, when hydrogen concentration in the solid solution exceeds hydrogen terminal solid solubility. In the present analysis, it is proven that hydrogen terminal solid solubility (TSS) in a metal takes the following form:

$$
\begin{aligned}
C^{T S} & =C_{e}^{T S} \exp \left(\frac{\bar{w}_{\text {int }}}{x R T}\right) \exp \left(\frac{W^{H}}{R T}\right), \\
\bar{w}_{\text {int }} & =-\int_{\mathbf{0}}^{\mathbf{E}^{\exp }} \int_{\bar{V}^{M}} \mathbf{S}^{a}: d \mathbf{E} d V_{0}, \\
W^{H} & =\left(\bar{V}^{H} / 3\right) \operatorname{tr}\left(\sigma^{a}\right) .
\end{aligned}
$$

The composition of the precipitating hydrides is given by $\mathrm{MH}_{x}$, where $\mathrm{M}$ could be any hydride forming metal and $x$ is the number of hydrogen moles in a mole of hydride. In relation (1a), hydrogen terminal solid solubility, $C^{T S}$, is defined on the reference configuration. $C_{e}^{T S}$, defined also on the reference configuration, is the terminal solid solubility, which is measured in experiments under conditions of chemical equilibrium and no externally applied stress. It is worth mentioning that $C_{e}^{T S}$ contains the effect of the energy of hydride accommodation, which is stored in the metal due to hydride expansion during precipitation; the energy of hydride accommodation is derived in section 3. $R$ and $T$ are the gas constant and the absolute temperature, respectively. $\bar{w}_{\text {int }}$, given in $(1 \mathrm{~b})$, is the interaction energy, which results from the interaction of the applied stress with the expanding hydride. The interaction energy is calculated by integrating over the molal volume of the metal, $\bar{V}^{M}$, in the nondeformed configuration, as well as up to the Lagrangian strain $\mathbf{E}^{\text {exp }}$, which describes stress-free hydride expansion. Generally $\bar{w}_{\text {int }}$ depends on the change of solid elastic properties during hydride precipitation [8,9]. However, in the present analysis, it is assumed, for simplicity, that the elastic properties of the hydride and the metal are identical. $W^{H}$ is the work per mole of addition of hydrogen in the solid solution, given by relation (1c), where $\operatorname{tr}\left(\sigma^{a}\right)$ is the trace of the applied Cauchy stress, $\sigma_{k k}^{a}$, and $\bar{V}^{H}$ is the molal volume of hydrogen in solid solution. Relations (1a) to (1c) lead to relations (8a) and (8b) of [15], under infinitesimal displacement gradient theory. They also lead to relations (9a) and (9b) of [9], under infinitesimal displacement gradient theory and 
identical hydride and solid solution elastic properties. The derivation of hydrogen terminal solid solubility follows.

Phase transformation is assumed to be a reversible process, which occurs under local chemical equilibrium among hydride, metal and hydrogen in solid solution:

$\mu^{h r}=\mu^{M}+x \mu^{H}$,

where $\mu^{h r}, \mu^{M}$ and $\mu^{H}$ are hydride, metal and hydrogen chemical potentials under stress, respectively, given in units of energy per mole (i.e. J/mol in SI units). The chemical potentials of mobile and immobile components in stressed solids have been derived in [5]. According to [5], the chemical potential of a component B in a solid, under stress, satisfies the following relation:

$\mu^{B}=\mu^{B, 0}+\frac{\partial w}{\partial N^{B}}-W^{B}$.

$\mu^{B, 0}$ is the chemical potential of component $\mathrm{B}$, under stress-free conditions, for the same concentration as that under stress. $w$ is the strain energy of the solid and $N^{B}$ is the number of $\mathrm{B}$ moles. Therefore, $\partial w / \partial N^{B}$ represents the strain energy of the solid per mole of component B. $W^{B}$ is the work performed by the applied stresses per mole of addition of component B. For immobile components, since the addition or removal of the component takes place at an external surface or an interface, the chemical potential is considered to be a surface property.

In the case of hydrogen, one may show, that $\partial w / \partial N^{H}$ is orders of magnitude smaller than $W^{H}$, given by relation (1c). Then, if $\partial w / \partial N^{H}$ is neglected, one derives that hydrogen chemical potential in a stressed solid takes the form $\mu^{H}=\mu^{H, 0}-\left(\bar{V}^{H} / 3\right) \operatorname{tr}\left(\sigma^{a}\right)$, which is identical to relation (7a) in [12]. $\mu^{H, 0}$ is assumed to follow the law for ideal or dilute solutions (Raoult's law).

By substituting (3), for every component, into (2) and using the law of ideal solutions for hydrogen, one may derive:

$$
x R T \ln \left(\frac{C^{T S}}{C^{T S, 0}}\right)=\left(\frac{\partial w}{\partial N^{h r}}-\frac{\partial w}{\partial N^{M}}\right)-\left(W^{h r}-W^{M}\right)
$$

$+x W^{H}$.

$C^{T S, 0}$ is the terminal solid solubility of hydrogen in the metal $\mathrm{M}$, under stress-free conditions. According to the discussion in section 4:

$$
\left(\frac{\partial w}{\partial N^{h r}}-\frac{\partial w}{\partial N^{M}}\right)-\left(W^{h r}-W^{M}\right)=\bar{w}_{a c c}+\bar{w}_{\text {int }},
$$

where $\bar{w}_{a c c}$ is the strain energy of hydride accommodation. Then by substituting (5) into (4) and taking into account the relation between stress-free terminal solid solubility and the experimental measurements:

$$
C_{e}^{T S}=C^{T S, 0} \exp \left(\frac{\bar{w}_{a c c}}{x R T}\right),
$$

relations (1a) to (1c), for hydrogen terminal solid solubility of a metal under stress, are derived.

In the following sections, the strain energy stored in the solid, when hydride precipitation occurs, is discussed and the mathematical model of hydrogen terminal solid solubility is further analyzed.

\section{ENERGY OF ACCOMMODATION OF A PRECIPITATING HYDRIDE}

A region of volume $\bar{V}^{M}$, in a stress-free metallic solid of total volume $V^{M}$, where $\bar{V}^{M} \ll<V^{M}$, is transformed into a hydride of volume $\bar{V}^{h r}$. This is the well-known transformation problem, which has been studied by Eshelby [16], in the case of infinitesimal displacement gradient theory. In order to relate to hydrogen terminal solid solubility, the volume of the transformed region is taken equal to the molal volume of the metal. The interface between the precipitating hydride and the surrounding metal is $S_{i}$. The geometry of the problem in the reference configuration, is given in Fig. (1). Hydride precipitation is associated with the stress-free expansion strain $\mathbf{E}^{\text {exp }}$, which depends on the metal. Because the hydride is constrained by the surrounding metal, during precipitation, a compressive stress field, $\mathbf{S}$, and an associated strain field, $\mathbf{E}$, develop, thus leading to the storage of elastic energy both in the precipitated hydride and the surrounding metal.

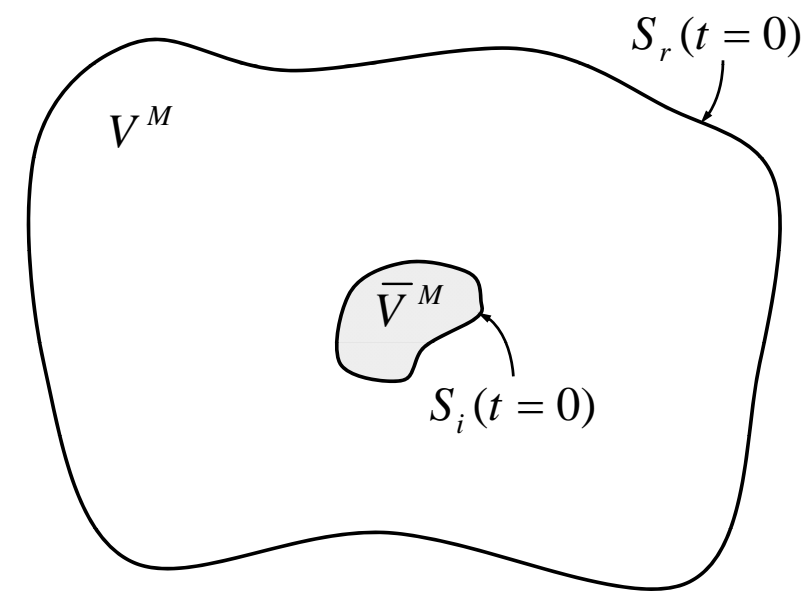

Fig. (1). Geometry of the hydride precipitation problem in the reference configuration $(t=0)$. The shaded area of the metal, of volume $\bar{V}^{M}\left(\bar{V}^{M} \ll V^{M}\right)$, transforms into a hydride. When the transformation occurs under stress, as in Section 4, traction is applied on the external boundary.

The differential of the elastic energy, in the transformed region, depends on the compressive stress field, $\mathbf{S}$, and the elasticity tensor, $\mathbf{K}$ :

$$
\begin{aligned}
& d w_{h r}=\int_{\bar{V}^{M}} \mathbf{S}: \mathbf{K}^{-1}: d \mathbf{S} d V_{0} \\
& =\int_{\bar{V}^{M}} \mathbf{S}:\left(d \mathbf{E}-d \mathbf{E}^{t r}\right) d V_{0},
\end{aligned}
$$


where $d V_{0}$ corresponds to the volume differential in the reference configuration. Also $d \mathbf{E}^{t r}$ is the differential of stressfree hydride transformation strain. On the reference configuration, the generalized Hooke's law is employed (e.g. [17]). Thus, the elasticity tensor relates linearly the differentials of the second Piola-Kirchhoff stress, $d \mathbf{S}$, and the total Lagrangian strain minus the stress-free expansion part, $d \mathbf{E}-d \mathbf{E}^{t r}$, i.e. $d \mathbf{S}=\mathbf{K}:\left(d \mathbf{E}-d \mathbf{E}^{t r}\right)$. Indeed, the principle of material frame indifference requires that, in terms of the second Piola-Kirchhoff stress, the response function of the material depends only on Lagrangian strain, or equivalently on Cauchy-Green deformation tensor $(\mathbf{C}=2 \mathbf{E}+\mathbf{I}$, where $\mathbf{I}$ is the identity tensor) or on the stretch tensor $\left(\mathbf{U}=\mathbf{C}^{1 / 2}\right)$, (e.g. [17]). Also the generalized Hooke's law, describing with sufficient accuracy the elastic response of metals, which are elastically stiff, has been used in several finite deformation models applied to various engineering problems (e.g. [18-22]).

The differential of the elastic energy, in the surrounding metal, satisfies the following relation:

$$
\begin{aligned}
& d w_{m}=-d t \int_{S_{i}(t)} \mathbf{n} \cdot \sigma \cdot \mathbf{v} d S=-d t \int_{\bar{V}^{h r}(t)} \sigma: \mathbf{D} d V \\
& =-\int_{\bar{V}^{M}} \mathbf{S}: d \mathbf{E} d V_{0} .
\end{aligned}
$$

$d V$ corresponds to the volume differential in the deformed configuration. $\mathbf{n}$ is the vector, normal to the interface, pointing from the hydride to the metal. Also $\mathbf{v}$ is the velocity of a solid particle on the interface and $\mathbf{D}$ is the respective rate of deformation tensor. In (8), relations $d \mathbf{E} / d t=\mathbf{F}^{T} \cdot \mathbf{D} \cdot \mathbf{F} \quad$ and $\quad \sigma: \mathbf{D} d V=\mathbf{S}:(d \mathbf{E} / d t) d V_{0} \quad$ were taken into account.

By adding relations (7) and (8) and integrating over hydride expansion strain, $\mathbf{E}^{\exp }$, one derives the energy of elastic hydride accommodation, in a metal under no externally applied stress:

$$
\bar{w}_{a c c}=-\int_{\mathbf{0}}^{\mathbf{E}^{\exp }} \int_{\bar{V}^{M}} \mathbf{S}: d \mathbf{E}^{t r} d V_{0} .
$$

In the case of infinitesimal displacement gradient theory, relation (9) leads to the well-known relation (2.21) of Eshelby [16]. When the metal is under stress, the strain energy of hydride accommodation is given by (15), where, from the total stress in the hydride, the externally applied part is subtracted.

\section{STRAIN ENERGY DURING HYDRIDE PRECI- PITATION UNDER STRESS}

In the present case, the metal is under externally applied Cauchy stress, $\sigma^{a}$, associated with a deformation gradient, $\mathbf{F}^{a}$, and a Lagrangian strain, $\mathbf{E}^{a}$. As in the previous section, a part of the volume, equal to molal volume of the metal in the reference configuration, $\bar{V}^{M}$, transforms into a hydride.

The elastic energy of the transformed region includes the strain energy, due to the applied stress, as well as the strain energy, due to constrained hydride expansion:

$$
\begin{aligned}
& w_{h r}=\int_{\mathbf{0}}^{\mathbf{S}^{a}} \int_{\bar{V}^{M}} \mathbf{S}: \mathbf{K}^{-1}: d \mathbf{S} d V_{0}+\int_{\mathbf{E}^{a}}^{\mathbf{E}^{h}} \int_{\bar{V}^{M}} \mathbf{S}: d \mathbf{E} d V_{0} \\
& -\int_{\mathbf{0}}^{\mathbf{E}^{\exp }} \int_{\bar{V}^{M}} \mathbf{S}: d \mathbf{E}^{t r} d V_{0} .
\end{aligned}
$$

In (10), $\mathbf{E}^{h}$ is the total Lagrangian strain, which develops in the hydride, during precipitation, and includes the transformation expansion strain $\mathbf{E}^{\exp }$.

The elastic energy in the metallic volume, which surrounds the hydride, satisfies the following relation:

$$
\begin{aligned}
& w_{m}=\int_{\mathbf{0}}^{\mathbf{S}^{a}} \int_{V^{M}-\bar{V}^{M}} \mathbf{S}: \mathbf{K}^{-1}: d \mathbf{S} d V_{0} \\
& -\int_{t(\mathbf{0})}^{t\left(\mathbf{E}^{\mathrm{exp}}\right)} d t \int_{S_{i}(t)} \mathbf{n} \cdot \sigma \cdot \mathbf{v} d S \\
& +\int_{t(\mathbf{0})}^{t\left(\mathbf{E}^{\mathrm{exp}}\right)} d t \int_{S_{r}(t)} \mathbf{n} \cdot \sigma^{a} \cdot \mathbf{v} d S .
\end{aligned}
$$

$S_{r}$ is the remote boundary of the metal, where $\sigma^{a}$ is applied. Both the interface between the transforming region and the surrounding metal, $S_{i}$, and the remote boundary, $S_{r}$, change with time, during transformation. Following a similar manipulation as in (8), one may show:

$$
\int_{t(\mathbf{0})}^{t\left(\mathbf{E}^{\exp }\right)} d t \int_{S_{i}(t)} \mathbf{n} \cdot \sigma \cdot \mathbf{v} d S=\int_{\mathbf{E}^{a}}^{\mathbf{E}^{h}} \int_{\bar{V}^{M}} \mathbf{S}: d \mathbf{E} d V_{0} .
$$

When (11) is added to (10) and (12) is taken into account, the total elastic energy in the metal and the hydride is derived:

$$
\begin{aligned}
& w=w_{m}+w_{h r}=\int_{\mathbf{0}}^{\mathbf{S}^{a}} \int_{V^{M}} \mathbf{S}: \mathbf{K}^{-1}: d \mathbf{S} d V_{0} \\
& -\int_{\mathbf{0}}^{\mathbf{E}^{\exp }} \int_{\bar{V}^{M}} \mathbf{S}: d \mathbf{E}^{t r} d V_{0}+\int_{t(\mathbf{0})}^{t\left(\mathbf{E}^{\exp }\right)} d t \int_{S_{r}(t)} \mathbf{n} \cdot \sigma^{a} \cdot \mathbf{v} d S .
\end{aligned}
$$

Relation (13) provides the strain energy of the solid, when a mole of metal transforms into a mole of hydride. Consequently, the sum of the last two terms on the righthand side of (13) is equal to the strain energy of the solid per mole of hydride minus the strain energy of the solid per mole of metal:

$$
\begin{aligned}
& \frac{\partial w}{\partial N^{h r}}-\frac{\partial w}{\partial N^{M}}=-\int_{\mathbf{0}}^{\mathbf{E}^{\exp }} \int_{\bar{V}^{M}}\left(\mathbf{S}-\mathbf{S}^{a}\right): d \mathbf{E}^{t r} d V_{0} \\
& -\int_{\mathbf{0}}^{\mathbf{E}^{\exp }} \int_{V^{M}} \mathbf{S}^{a}: d \mathbf{E}^{t r} d V_{0} \\
& +\int_{t(\mathbf{0})}^{t\left(\mathbf{E}^{\exp }\right)} d t \int_{S_{r}(t)} \mathbf{n} \cdot \sigma^{a} \cdot \mathbf{v} d S .
\end{aligned}
$$

The first integral of the right-hand side is equal to the strain energy of hydride accommodation, taking into account that in the present case the metal is under externally applied stress, $\mathbf{S}^{a}$ :

$$
\bar{w}_{a c c}=-\int_{\mathbf{0}}^{\mathbf{E}^{\exp }} \int_{\bar{V}^{M}}\left(\mathbf{S}-\mathbf{S}^{a}\right): d \mathbf{E}^{t r} d V_{0} .
$$

Also the third integral of right-hand side of (13) and (14) is equal to the work per mole of addition of hydride minus 
the work of addition of a mole of metal, both performed by the applied stress:

$W^{h r}-W^{M}=\int_{t(\mathbf{0})}^{t\left(\mathbf{E}^{\mathrm{exp}}\right)} d t \int_{S_{r}(t)} \mathbf{n} \cdot \sigma^{a} \cdot \mathbf{v} d S$.

Therefore, by taking into account relations (14), (15), and (16) as well as (1b), where $\bar{w}_{\text {int }}$ is defined, one derives relation (5).

\section{STRESS EFFECT ON HYDRIDE PRECIPITATION}

Two cases of hydride precipitation under simple homogeneous deformation fields, namely dilatation and simple shear, are investigated in the present section. In both cases the hydride is assumed to expand isotropically, while precipitating. Therefore the Lagrangian expansion strain is related to the ratio of molal volumes of hydride and metal as follows:

$\mathbf{E}^{\exp }=\frac{1}{2}\left[\left(\frac{\bar{V}^{h r}}{\bar{V}^{M}}\right)^{2 / 3}-1\right] \mathbf{I}$.

In the case of dilatation, the spatial and material coordinates of a metal particle satisfy the following relations (e.g. [23]):

$\mathbf{x}=\left(\frac{2}{3} \frac{S_{D}}{K}+1\right)^{1 / 2} \mathbf{X}$

where $K$ is the bulk modulus of elasticity of the metal. Also $S_{D}$ is the hydrostatic stress, applied on the reference configuration:

$\mathbf{S}^{a}=S_{D} \mathbf{I}$.

After straightforward calculations, one may show that the work per mole of addition of hydrogen in the solid solution and the interaction energy depend also on hydrostatic stress:

$W^{H}=\frac{S_{D} \bar{V}^{H}}{\sqrt{\frac{2}{3} \frac{S_{D}}{K}+1}}$,

$\bar{w}_{\text {int }}=-\frac{3}{2} S_{D} \bar{V}^{M}\left[\left(\frac{\bar{V}^{h r}}{\bar{V}^{M}}\right)^{2 / 3}-1\right]$.

Relations (20) and (21) reveal opposite effects of hydrostatic stress on hydrogen terminal solid solubility. Increase of hydrostatic stress leads to decrease of terminal solid solubility, through the contribution of the interaction energy. Indeed a hydrostatic tension field facilitates energetically the transformation of an expanding particle. On the other hand, increase of hydrostatic stress leads to increase of terminal solid solubility, through the contribution of the work per mole of addition of hydrogen in the solid solution. This is also an expected trend, since a hydrostatic tension field creates space for hydrogen atoms, thus favoring hydrogen in solid solution. The overall effect is given in Fig. (2) for Zircaloy- 2 and $\delta$-hydride $\left(\mathrm{ZrH}_{1.66}\right)$ at $573 \mathrm{~K}$. Zircaloy-2, a hy- dride forming alloy with properties presented in Table $\mathbf{1}$, is used in the fuel cladding of nuclear reactors, where the temperature under consideration develops. The sources of material properties are given in [7].

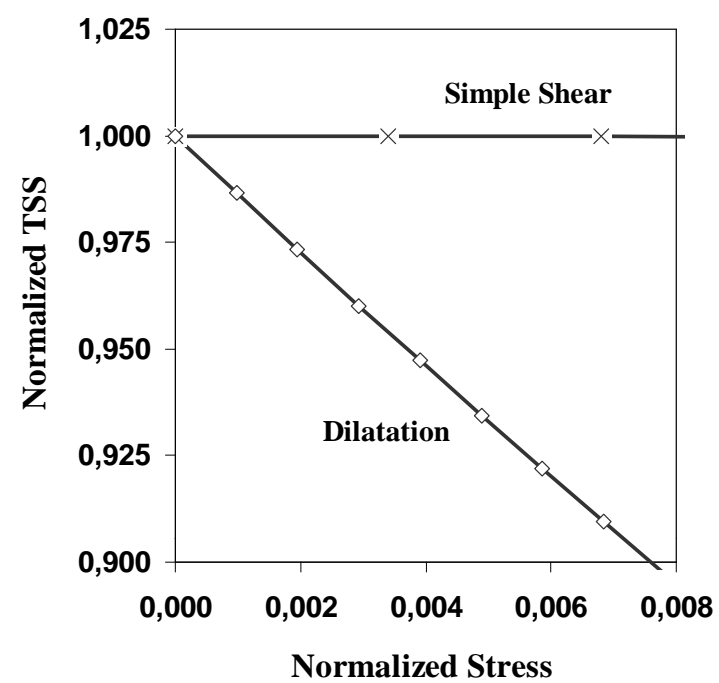

Fig. (2). Effects of applied stress on normalized hydrogen terminal solid solubility, $C^{T S} / C_{e}^{T S}$, of Zircaloy-2 at $573 \mathrm{~K}$, in the case of dilatation and simple shear, where the normalized stress is given by $S_{D} / K$ and $S_{S} / \mu$, respectively.

Table 1. Material Properties of Zircaloy-2 and $\delta$-Hydride $\left(\mathrm{ZrH}_{1.66}\right)$ at $573 \mathrm{~K}$

\begin{tabular}{|c|c|}
\hline$K$ & $102.3 \mathrm{GPa}$ \\
\hline$\mu$ & $29.4 \mathrm{GPa}$ \\
\hline$\lambda$ & $82.7 \mathrm{GPa}$ \\
\hline $\bar{V}^{M}$ & $14 \cdot 10^{-6} \mathrm{~m}^{3} / \mathrm{mol}$ \\
\hline $\bar{V}^{h r}$ & $16.3 \cdot 10^{-6} \mathrm{~m}^{3} / \mathrm{mol}$ \\
\hline $\bar{V}^{H}$ & $7 \cdot 10^{-7} \mathrm{~m}^{3} / \mathrm{mol}$ \\
\hline$x$ & 1.66 \\
\hline
\end{tabular}

According to Fig. (2), hydrogen terminal solid solubility decreases by more than $10 \%$, when hydrostatic stress exceeds the yield stress of irradiated alloy, which is equal to $580 \mathrm{MPa}$ at $300^{\circ} \mathrm{C}$ (e.g. [9]).

In the case of simple shear, the spatial and material coordinates of a metal particle satisfy the following relations (e.g. [23]):

$x_{i}=\delta_{i J} X_{J}+\left(\frac{S_{S}}{\mu}\right) \delta_{i 1} X_{2}$,

where small and capital letter indices are used to distinguish between spatial and material coordinates. $\delta_{i J}$ is Kronecker delta, $\mu$ is the shear modulus of the metal and $S_{S}$ is the shear stress applied on the reference configuration. The ten- 
sor of the applied stress includes also higher order normal components:

$$
\mathbf{S}^{a}=\left[\begin{array}{ccc}
\frac{1}{2} \lambda\left(\frac{S_{S}}{\mu}\right)^{2} & S_{S} & 0 \\
S_{S} & \left(\frac{1}{2} \lambda+\mu\right)\left(\frac{S_{S}}{\mu}\right)^{2} & 0 \\
0 & 0 & \frac{1}{2} \lambda\left(\frac{S_{S}}{\mu}\right)^{2}
\end{array}\right] .
$$

In the case of simple shear, the work per mole of addition of hydrogen in the solid solution and the interaction energy depend on applied shear stress, according to the following relations:

$$
\begin{aligned}
& W^{H}=\left(\frac{1}{2} \lambda+\mu\right) \bar{V}^{H}\left[\left(\frac{S_{S}}{\mu}\right)^{2}+\frac{1}{3}\left(\frac{S_{S}}{\mu}\right)^{4}\right], \\
& \bar{w}_{\text {int }}=-\frac{3}{4} K\left(\frac{S_{S}}{\mu}\right)^{2} \bar{V}^{M}\left[\left(\frac{\bar{V}^{h r}}{\bar{V}^{M}}\right)^{2 / 3}-1\right] .
\end{aligned}
$$

According to relations (24) and (25), the effect of applied shear stress on hydrogen terminal solid solubility is weak, involving second and fourth order terms of $S_{S} / \mu$. Numerical results are given in Fig. (2) for Zircaloy- 2 and $\delta$-hydride.

The strong effect of hydrostatic stress on hydrogen terminal solid solubility has direct implications on delayed hydride cracking. Indeed the area of large hydrostatic stress is directly ahead of the tip of an open mode crack, on the crack plane. In this area, hydrogen terminal solid solubility decreases most and the precipitation of brittle hydrides is facilitated. As a consequence the crack propagates ahead without deviation from the crack plane. Experimental studies on Zirconium alloys show, in the presence of hydrogen, the development of long straight cracks with fractured hydrides along the crack faces (e.g. fig. 5-4 in [11]). Also due to crack tip blunting hydrostatic stress is maximum at a distance from the crack tip (e.g. [21, 24]). Therefore the hydrides precipitate at distance from the crack tip, which is also confirmed by experimental studies (e.g. [10]).

\section{CONCLUSIONS}

In the present study, the terminal solid solubility of hydrogen in a stressed metal is derived, analytically, based on finite elasticity. The phase transformation is considered to be a reversible process, occurring under local chemical equilibrium among hydride, metal and hydrogen in solid solution. It is shown that, besides temperature, hydride precipitation depends on stress due to (i) the interaction energy per mole of transforming metal, which results from the interaction of the applied stress field with the field of the expanding hydride and (ii) the work of addition of a mole of hydrogen in the metal, which also measures the reduction of hydrogen chemical potential in solid solution, caused by hydrostatic stress. The stress effect due to the variation of the elastic strain energy of the metal per mole of hydrogen in solid solution is neglected. The finite deformation relations have a structure similar to the structure of previous relations, derived based on infinitesimal displacement gradient theory [9, 15], and lead to them in the small strain limit. The effects of dilatation and simple shear on hydrogen terminal solid solubility are also investigated. It is shown that hydrostatic stress strongly influences hydrogen terminal solid solubility, which agrees with experimental observation on position of hydride precipitation ahead of a mode-I blunted crack and on direction of crack growth. The present analysis is complementary to previous papers on mass diffusion and non-mechanical energy flow in metals under finite deformation $[12,13]$ and it is expected to facilitate hydride induced embrittlement studies under conditions, which require finite deformation approach.

\section{REFERENCES}

[1] Takano S, Suzuki T. An electron-optical study of $\beta$-hydride and hydrogen embrittlement of vanadium. Acta Metall 1974; 22: 26574.

[2] Birnbaum HK, Grossbeck ML, Amano M. Hydride precipitation in $\mathrm{Nb}$ and some properties of $\mathrm{NbH}$. J Less-Common Metals 1976; 49: 357-70.

[3] Grossbeck ML, Birnbaum HK. Low temperature hydrogen embrittlement of niobium II - microscopic observations. Acta Metall 1977; 25: 135-47.

[4] Shih DS, Robertson IM, Birnbaum HK. Hydrogen embrittlement of $\alpha$ titanium: in situ TEM studies. Acta Metall 1988; 36: 111-24.

[5] Li JCM, Oriani RA, Darken LS. The thermodynamics of stressed solids. Z Phys Chem 1966; 49: 271-90.

[6] Lufrano J, Sofronis P, Birnbaum HK. Modeling of hydrogen transport and elastically accommodated hydride formation near a crack tip. J Mech Phys Solids 1996; 44: 179-205.

[7] Varias AG, Massih AR. Hydride-induced embrittlement and fracture in metals - Effect of stress and temperature distribution. J Mech Phys Solids 2002; 50: 1469-510.

[8] Varias AG. Effects of hydride elastic properties on hydrogen terminal solid solubility and sub-critical crack growth in metals. In: Proceedings of the 7th National Congress on Mechanics; Chania, Greece, 2004.

[9] Varias AG, Feng JL. Simulation of hydride-induced steady-state crack growth in metals - Part I: growth near hydrogen chemical equilibrium. Comput Mech 2004; 34: 339-56.

[10] Huang FH, Mills WJ. Delayed hydride cracking behavior for ZIRCALOY-2 tubing. Metall Trans A 1991; 22A: 2049-60.

[11] Efsing P. Delayed Hydride Cracking in Irradiated Zircaloy. Royal Institute of Technology. Stockholm 1998.

[12] Varias AG. On the diffusion in solids under finite deformation. Open Mech J 2007; 1: 26-8.

[13] Varias AG. On the coupling of mass diffusion and non-mechanical energy flow in metals under finite deformation. Open Mech J 2008; 2: $57-61$.

[14] Lee JK, Earmme YY, Aaronson HI, Russell KC. Plastic relaxation of the transformation strain energy of a misfitting spherical precipitate: ideal plastic behavior. Metall Mater Trans 1980; A11: 183746.

[15] Varias AG. Hydrogen embrittlement and sub-critical crack growth in hydride forming metals. J Mech Behav Mater 2005; 16: 211-39.

[16] Eshelby JD. The determination of the elastic field of an ellipsoidal inclusion and related problems. Proc R Soc Lond 1957; A241: 37696.

[17] Malvern LE. Introduction to the mechanics of a continuous medium. Englewood Cliffs, New Jersey: Prentice-Hall 1969.

[18] Harren SV, Asaro RJ. Nonuniform deformations in polycrystals and aspects of the validity of the Taylor model. J Mech Phys Solids 1989; 37: 191-232.

[19] Povirk GL, Needleman A, Nutt SR. An analysis of residual stress formation in whisker-reinforced Al-SiC composites. Mater Sci Eng 1990; A125: 129-40.

[20] Varias AG, Massih AR. Temperature and constraint effects on hydride fracture in zirconium alloys. Eng Fract Mech 2000; 65: 29. 54.

[21] Varias AG, Suo Z, Shih CF. Ductile failure of a constrained metal foil. J Mech Phys Solids 1991; 39: 963-86. 
[22] Mohan R, Ortiz M, Shih CF. An analysis of cracks in ductile single crystals - I: anti-plane shear. J Mech Phys Solids 1992; 40: 291-

$$
313 .
$$

[23] Atkin RJ, Fox N. An introduction to the theory of elasticity. Longman: London 1980.
[24] McMeeking RM. Finite deformation analysis of crack-tip opening in elastic-plastic materials and implications for fracture. J Mech Phys Solids 1977; 25: 357-81.

(c) Andreas G. Varias; Licensee Bentham Open.

This is an open access article licensed under the terms of the Creative Commons Attribution Non-Commercial License (http://creativecommons.org/licenses/by-nc/3.0/) which permits unrestricted, non-commercial use, distribution and reproduction in any medium, provided the work is properly cited. 